(REview ARTiCLE)

\title{
Cryptographic ledger of blockchain technology in healthcare
}

Diba Chowdhury, Laila Tamanna Anni, Md. Mahmudul Hasan, Nayeem Al-Tamzid Bhuiyan *, Nafiul Islam and Rahat Pervez

Department of Electrical and Computer Engineering, North South University, Dhaka - 1229, Bangladesh.

GSC Advanced Research and Reviews, 2021, 07(03), 028-037

Publication history: Received on 04 May 2021; revised on 10 June 2021; accepted on 12 June 2021

Article DOI: https://doi.org/10.30574/gscarr.2021.7.3.0117

\begin{abstract}
Blockchain technology produced a unique nature called Public Health Record (PHR) to shared medical-related data between clients. The big achievement of this technology there is no need for any third party and user can control their personal data. This technology offers encrypted data, integrated data, drug traceability, interoperability and most important thing it gives security regarding patients' data misuse, drug counterfeit. It is distributed framework, maintenance cost, improved our medical sector.
\end{abstract}

Keywords: Distributed networks; Smart B2B contract; Automation; Security

\section{Introduction}

Blockchain is a decentralized, distributed database, peer-to-peer network, digital ledger and allowed to history, blockchain promoted new physiognomy called electronic health records. Blockchain can connect multiple computers by nodes and it has no transaction create new blocks that help to pass secure information from one person to another person. In medical cost. Client can access all the authenticated and verifiable medical-related information through blockchain which is secured by cryptography. Anyone can select transactions and also added new chain in block. The master key of blockchain is hash, and via this hash function blockchain can create a unique id to cryptocurrency to added data. As an example, bitcoin is the first cryptocurrency and it creates a proof of work system method. These methods working to validate the block validator or miner with handle the ploy. Basically, this is a competition and winner win's according to speed of creating block using hash function and conqueror deposited for his/her duty with a short amount of getting. On the other hand, blockchain provides cryptocurrency transactions and through this data can encode into hash and there is a chance to anyone mentioned different ideas. Resources which are called HL7 Fast Healthcare Interoperability Resources (FHIR) proposed by MIT and Mayo Clinic developed control of patients' information granted to access and also exchange information. A group of China also created an app which is also developed health view and directly access health records of patients and this process is called Healthcare Data Gateway (HGD) [1].

\section{Principle/Issues of blockchain technology in healthcare}

In order to establish transparency and trust blockchain represents a peer-to-peer distributed ledger technology for a new generation of transactional applications. The underlying fabric of bitcoin is blockchain. It has three main components of design patterns. These are- A Distributed Network, A shared Ledger and Digital Transaction.

\subsection{Distributed networks}

The blockchain is a peer-to-peer decentralized architecture with nodes consists of network participants.

\footnotetext{
* Corresponding author: Nayeem Al-Tamzid Bhuiyan

Department of Electrical and Computer Engineering, North South University, Dhaka - 1229, Bangladesh. 
Every block of the chain stores an identical copy of the blockchain. It takes part in the collective process of validating and it also ensures the certified digital transaction of the network. Distributed networks mainly work to connecting thousands of devices so that in health care system patients and doctors easily interact with each other within few minutes. Via this network administrator has control power which is called central mode and this system also provides autonomy.

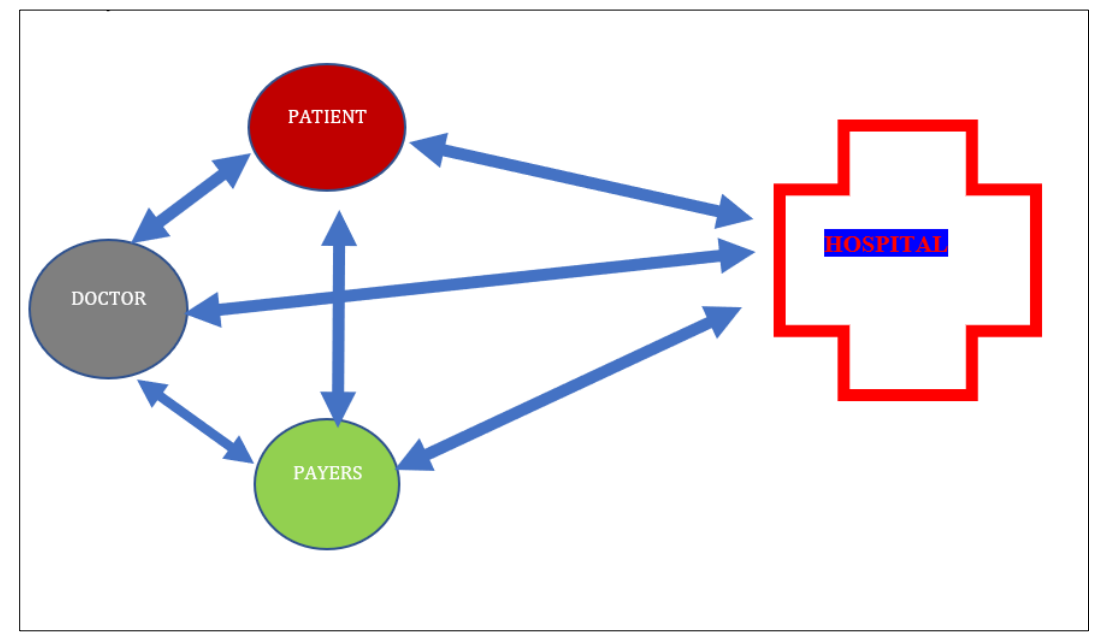

Figure 1 Distributed network work in healthcare

\subsection{Shared ledger}

It is a distributed database that is shared, consensus of replicated and synchronized many sites, geographies, etc. The underlying of shared ledger technology is the blockchain and which is also the technology that underlies bitcoin. The distributed network members record digital transactions into a shared ledger. The member in the network runs an algorithm to evaluate adding transactions. When the majority of the members agree that the transaction is valid only then new transaction can be added to the shared ledger. The new change of the ledger is reflected in all the copies of the blockchain in minutes or in some cases seconds. After the addition of the new transaction, it cannot be changed or removed. As all the members have a copy of the blockchain so, no single member can change the new one or remove it.

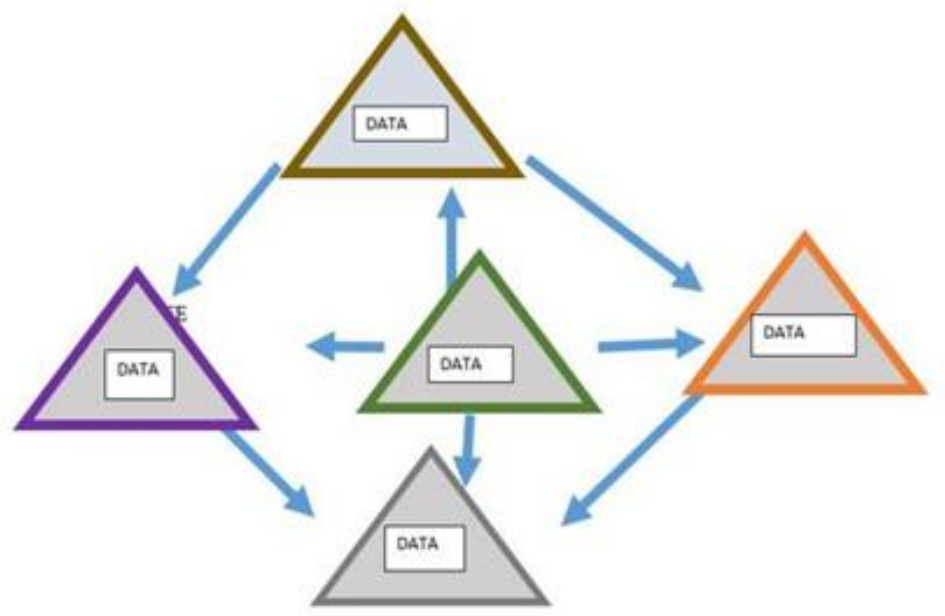

Figure 2 Structure of shared ledger

\subsection{Digital transaction}

Digital transaction helps people to move money directly one to another through blockchain. In digital transactions there is no central point, multiple nodes can be connected. It is allowed to transfer initiative amount to recipient's bitcoin address using same network. Blockchain work in synchronous order so that blocks are occupied chronologically. First 
of all initial transaction data packaged in a block. Secondly, block transmitted to members. After consensus and approval by network block added to the chain then the update is distributed.

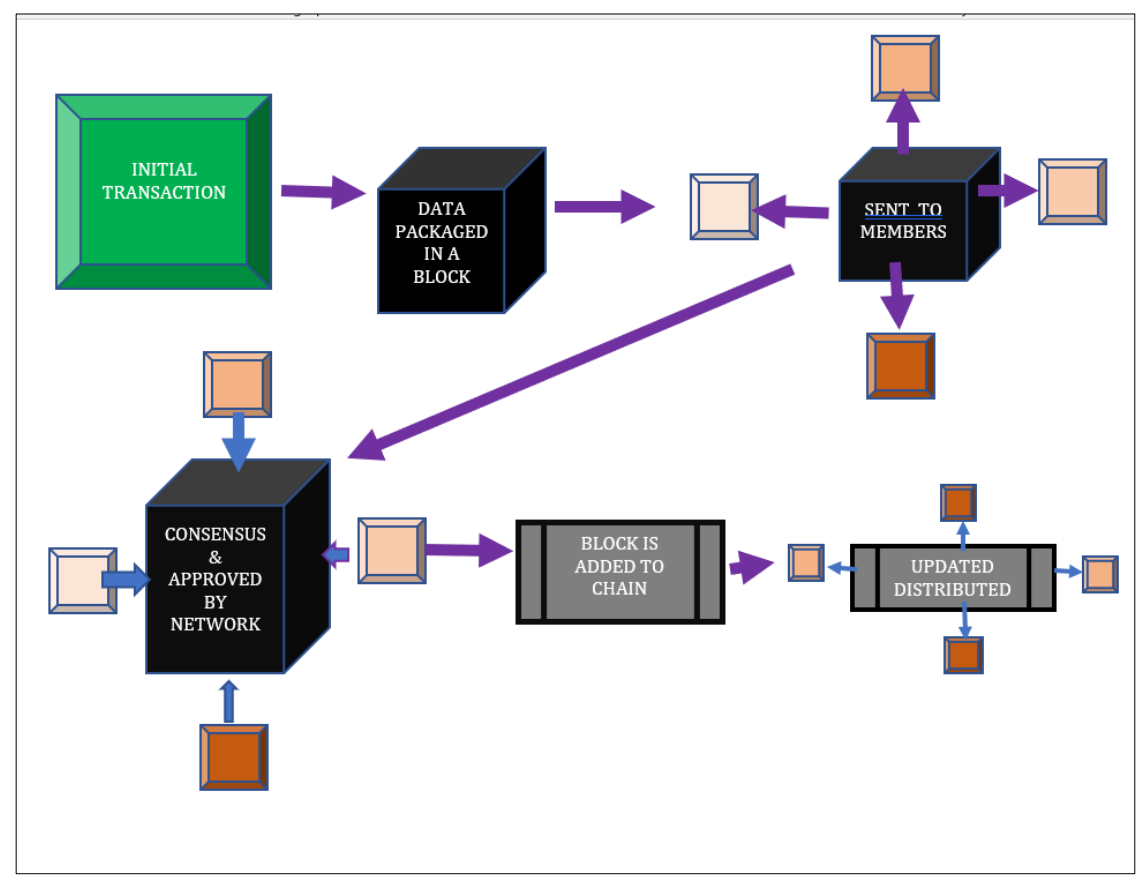

Figure 3 How digital transaction work

\section{Applications of blockchain technology}

In today's world, blockchain is a magical word. Initially, the purpose was to build a transparent platform for digital transactions.

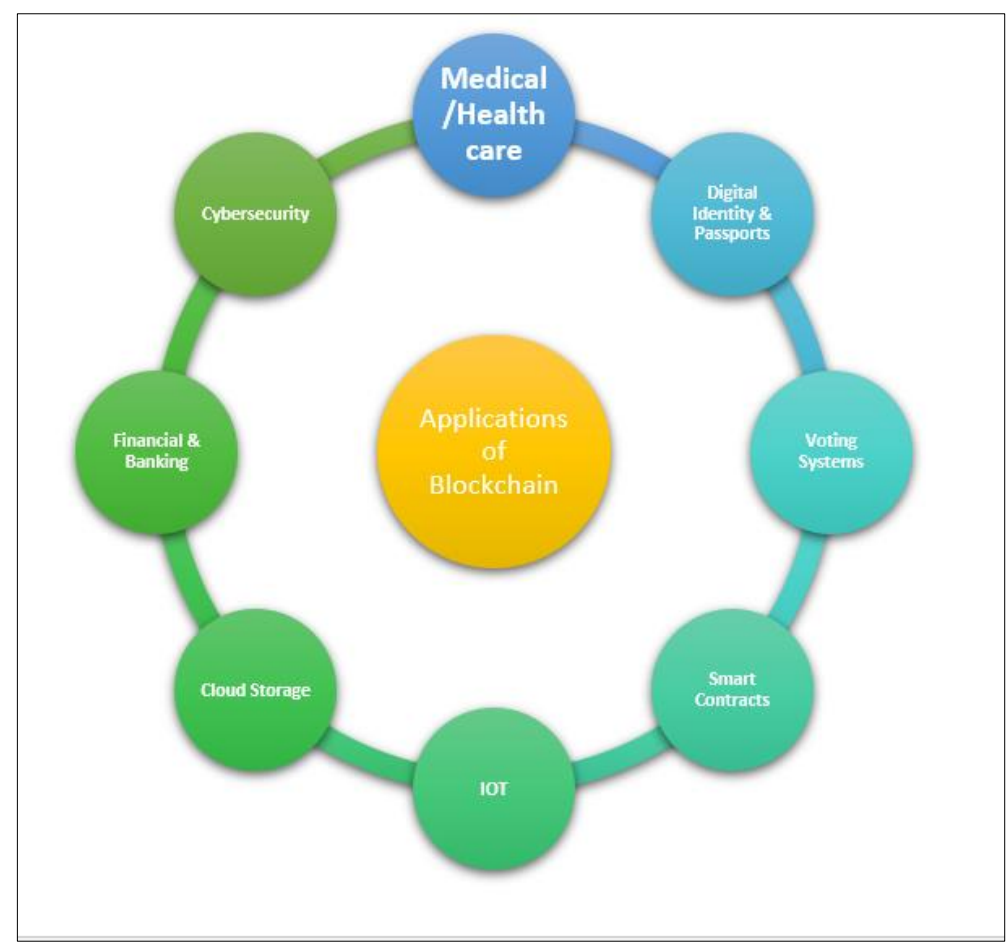

Figure 4 Applications of blockchain 
But now this cutting-edge technology has spread the magic all over the industry. Every different kind of industry are now using application based on blockchain for completing their daily task like online shopping, ticket booking, doctor's appointment, money transaction, service provider, id validation, vote casting, banking, money lending, making micropayment, paying parking fines, car renting, buying drinks, etc. Healthcare sector also uses blockchain technology to optimize its management system as well as to secure the system. Already there is a system based on blockchain, named Matapedia, which gives a patient all kind of information about treatment, verified doctors, hospitals, etc. There are already so many significant applications in various sectors for blockchain-like Smart Contracts, Ethereum, Grid coin, Ripple Labs, etc.

\section{How can blockchain technology transfer the healthcare sector?}

Supply chain, medical data sharing, fraud, drugs problem, management, etc. These are challenging problems in healthcare sector. So, investigating solutions to those problems blockchain technology entered in healthcare sector. Supply chain is a big factor in healthcare sector because patients' safety, risk, security, integrity, complexity, data provenance, health outcomes depend on this factor. And blockchain technology prevents all the risks and provides efficient secured patients' medical history. Blockchain technology gives tidings of client and also established all the source of payers, stake- holders that's why there is no chance of double-dealing. Blockchain technology also secured that validation of payment process. Using reliability-centered maintenance (RCM) operation blockchain technology able to ameliorate running information like processing time, collaboration shows, payment method, reduce cost. Blockchain technology monitoring the real-time information of drugs and shows the transaction of pharmaceutical products. In this way, blockchain technology disposes of the healthcare sector. Blockchain technology plays important role in the healthcare sector. Through massive electronic smart records, healthcare sector currently improved communication between patients and doctors. Blockchain technology permitted that third party can ingress information transparency and it is very beneficial in emergency cases. Blockchain offers peer to peer network of transactions and this network check all information and verified that information is right or wrong. If the information is right then it is stored in the block. Using consensus algorithm in blockchain which is helpful to create chain of block. Interoperability is one of the most important features of blockchain technology. This feature allowed exchanged health-related in- formation between different hospitals, managed data, store information, shared patient-related information so that it can provide a reliance relationship between healthcare communities [2].

\section{Reducing the cost of verification and networking}

In an era of emerging technologies, when health care budget and peoples need are constantly increasing, blockchain technology came to us as the best solution for the betterment of health system and hospitals, making sure by reducing costs that will lead us to the better outcomes of patients.

\subsection{Credentials verifications for physicians}

Blockchain technology has the power to cut the cost up to 80 percent and the time it requires to complete the credentials verifications process of physicians. At present, a physician usually sends their credential application to several organizations for the verification process which may take long months to complete. However, with the blockchain technology currently on the scene, members can easily access physician encrypted credentials. Moreover, changing the credentials of physicians is not possible without validating it by the members of the network. A physician also can share their access code with the hospital for reviewing their credentials. So, it's a better option to put the trust in blockchain technology other than a third party.

\subsection{Payment processing}

One of the intriguing advantages of blockchain in healthcare is we don't have to use cash for payment because of the presence of cryptocurrencies. Thus, we can eliminate fraud and unperformed billing services. Blockchain technology can easily detect fraud money. Currently, we need a third party for automation bill process but blockchain will remove that and so will reduce the total organizational cost. However, payment process through cryptocurrencies will have a revolutionary impact in the healthcare industry. Each payment performed would be tracked which will make sure eliminating the fraud in the process. Bitcoin is the platform where many of the blockchain-based payment processing exists. Suppose a client wants to send money to B client. Through bitcoin any international payment processing occurs easily within one to three days. The internal process is first of all local currency of a client converted into bitcoin then this bitcoin converted into local currency of client B. So that a client is payers and B client is receiver. More of the company likes Kenya, Bites used the bitcoin method and provides a faster and cheaper way to the client and as well for company. 


\subsection{Security}

Currently, a single central database managed by one entity holds all the information regarding the health care system and organization, which is very risky. If someone hacks the system, he/she will easily get it all in one single point. But blockchain can prevent this type of internal failure of an organization and can provide more security and reliability. In a blockchain ledger, an organization will have multiple levels of access which will be encrypted. Thus, blockchain will help to prevent the attack of hackers, hardware failure and data corruption. Blockchain using many mechanisms like cryptography, models of behavior and decision making, supply chain management, game theory so that it provides highlevel security, hashing. Hashing mainly creates the path between payers and receivers, received the input and provides the output. Input and output size must be same. Any changes affected the input and output. If input is change then output is also changing. On the other side cryptography paired the public and private keys. Private Key needs authentication and users have power to control, converted into digital transaction. Game theory is using for range-related purpose. Satoshi Nakamoto generated the framework-related bitcoin and it is a costly resource-intensive process. It is working by proof of work (pow) algorithm so include mining node and consists of money and time. So that it has capability to find who is the node and where did this mining node come from. Any dishonest person propelled because of its strong trace feature.

\section{Payers/consumers and providers}

Payers are mainly insurance providers and trying to convince patients, healthcare potential and also provides immune payment process, untroubled electronic data interchange (EDI) via digital system. Currently, IBM propagated that blockchain deal with different insurance company including healthcare service corporation, PNC bank, Anthem and Aetna. Micropayments is another thing like Cigna Healthy Rewards Pro-gram that explained as connected people with this connotation gets discount if he/she signs up for checkups like health-related thing, vision and hearing care, fitness or buying healthcare-related products [3].

\subsection{Medical history}

Nowadays in medical section we find the patients' different information, medical his- tory is in hotchpotch situation. Using decentralized medication management system (DMMS) we are able to sort all information in synchronized way. Suppose doctor recommend a patient to another doctor for treatment purpose then patients need to carry all reports, all information but DMMS helps to transfer all of the histories, information to that doctor. It is the most interesting thing about blockchain. This blockchain technology mainly created a medical chain that boosts metastasis information securely, immutable and transparently manner. Every member can see all of the changes in a blockchain and also has a way to check the data so that the full thing happened in a transparent way. This technology is also able to detect fraud. In pharmaceutical blockchain helps to sort the data and we all know that pharmaceutical mainly consists of large data, deal with analytical data report, and store the data. Blockchain is showing the platform as AI to safely store and sort thousands of datas. Pharmacy companies can save their money using blockchain technology and it is also secured, protected because it has the capability to stored information of all the payers, consumers, and clients. If every pharmaceutical company, hospital and also different source like any organization entered into this supply chain then we can save our money, time, and able to create a secured path, risk-free, effective. Via blockchain we are able to manage health supply chain without any loss also in a protected way.

\subsection{Sell your medical data on a blockchain marketplace}

Via blockchain technology researchers are searching right medical data and also looking for right patient. So in blockchain marketplace basically researchers are selling the right information to access the right patient. Emblem is a blockchain-based app that basically doing collects information about pharmacy and also selling information to pharmacy and grant a patient selling their proper data to anyone who wants to purchase it. The White Paper introduced by consortium of healthcare players and the banner of EY. This method has two models. One is altruistic and another one is incentive. On the altruistic platform, it is allowed to share all medical data. On the other hand, incentive provides to sell and buy the data in a secure way. Blockchain technology gives that much flexibility that anyone can track what buyer/seller does with data. This process is called tokenization. As an example, telemedicine marketplace is created using blockchain-generated start-ups with known payers and sell their data/buy their data. This is the zone where people get profit, save time and also able to create blueprint of healthcare. The major part if anyone sells/buys data set already and after some time he/she wants to change something blockchain technology gives you the power to control your data. 


\section{Implementation of blockchain technology in healthcare}

\subsection{Consent management}

It has been a long time since the sector of clinical research seen any development. Its facility is limited in case of providing best possible solutions in the field of healthcare services. Especially clinical trials need improvement in terms of quality. For getting real-time results from patients, it is absolutely necessary to increase the consent management of rights to be using data in clinical trials. Basically, patient consent means letting the patient know about all the steps and process that needs to be done in clinical trials and the risk in it. The clinical trial has four main phases of consent. First phase determines the side effect of drugs in the human body. Second phase determines the treatment efficiency by keeping people in observation. Third phase is bigger testing of close examination where large scale number of patients is needed. This phase gives the approval for marketing. Fourth phase is after marketing supervision. The biggest problem in all these is people don't want to consent to their information mostly because of lack of trust, reliability and fear of misuse. Nowadays, medical information is more valuable than credit card information in the black market. By knowing someone's medical info, one can know all about a person's lifestyle, genes and identities. Some high potential attacks by targeting patients' sensitive medical information happened in the past years. That's why people now feel more sensitive about sharing their medical data. Besides, for analysis part, patients have to fill up so many forms which are very time-consuming and discouraging. That's another reason normal people feel less interested in participating. Most sick people participate in clinical trials [4]. Consent management is the main challenge here. People have close to no control over how their data is shared among researchers, pharmaceutical company and medical institutions. The security in clinical trials for data storing and distribution is very poor. People need more assurance that their data are secured and protected. Blockchain technology gives effective solutions to all these clinical trial problems. Blockchain can implement protocols in terms of collecting, tracking and storing the consent through cryptographic encryption. After conducting a transaction on a network, all the data are recorded and validated through cryptography. Thus, it won't be possible to alter anything which will improve transparency and data integrity. This validation system can make people willingly share their consent. Also, people will have more control over their data now. With blockchain in market, people are now the center of this ecosystem. A unique master document named Chain script holds all the patients' data as a distinct structure. Hash encrypts every single data in Chain script. Nobody except authorized one can access those data. Thus, blockchain improves the consent data management system. A hospital in France has already developed a platform like this where patient can verify their consent registration by their unique signature only. Also, there is MIT's Enigma. It is also a secured platform for clinical research.

\subsection{Counterfeit medicine and blockchain}

In today's world, counterfeit drugs are million-dollar problem in the healthcare industry. According to The World Health Organization, $10 \%$ of the medicines sold across the world is fake and in low-income countries, the percentage is somewhat higher. The alarming news is that the counterfeit market is valued at almost $\$ 200$ yearly. WHO estimates that these fake drugs have around $16 \%$ wrong ingredients while $17 \%$ have the wrong levels of active ingredients? These fake medicines do not have the ability to treat the diseases which can put a patient's life at risk. Aside from the fact that it causes the patients some serious health damage, counterfeits also bring tremendously financial loss for the pharmaceutical industry every year [4]. Blockchain technology can make the counterfeit problem a non-issue. Not only can it keep the track of the entire supply chain of the drug, but it also offers recovery. Whenever a drug shipment goes missing, with the help of blockchain technology, we can trace all the parties that were involved in the drug sharing process. Blockchain technology has made it possible to keep track, trace and verify drugs at the same time at every level of supply chain, from the pharmaceutical to all the way to the patients. No data alteration is possible with this technology. All the data are permanent after adding to the block. Blockchain stores information about each movement of drugs among the entities. So, if something happens, labs or stakeholders can identify the exact location of the problem and solve them immediately.

\subsection{Smart B2B contract}

Smart b2b contracts with blockchain are not only decentralized but also provide automation. For security purposes, all information will be saved after including in the smart contract. Through smart B2B contract, anyone can shuffle money, substance, valuable things. In health sector smart contract is working as bridge between patient, doctor, consumers, and industry. As an example, Smart contract basically collects the information of verified doctors, patients and only permitted clients can access the information. The interesting thing is smart contracts help to create agreements without need for any third party. In smart contract, there is a backup option so that client can access same thing after deleting. This has happened because blockchain created copy things multiple times over. This is also helping us to save our money because we don't need any third party, so we don't need to pay anyone for creating law or agreement. We can deal with it directly via smart contract on the blockchain platform. 
GSC Advanced Research and Reviews, 2021, 07(03), 028-037

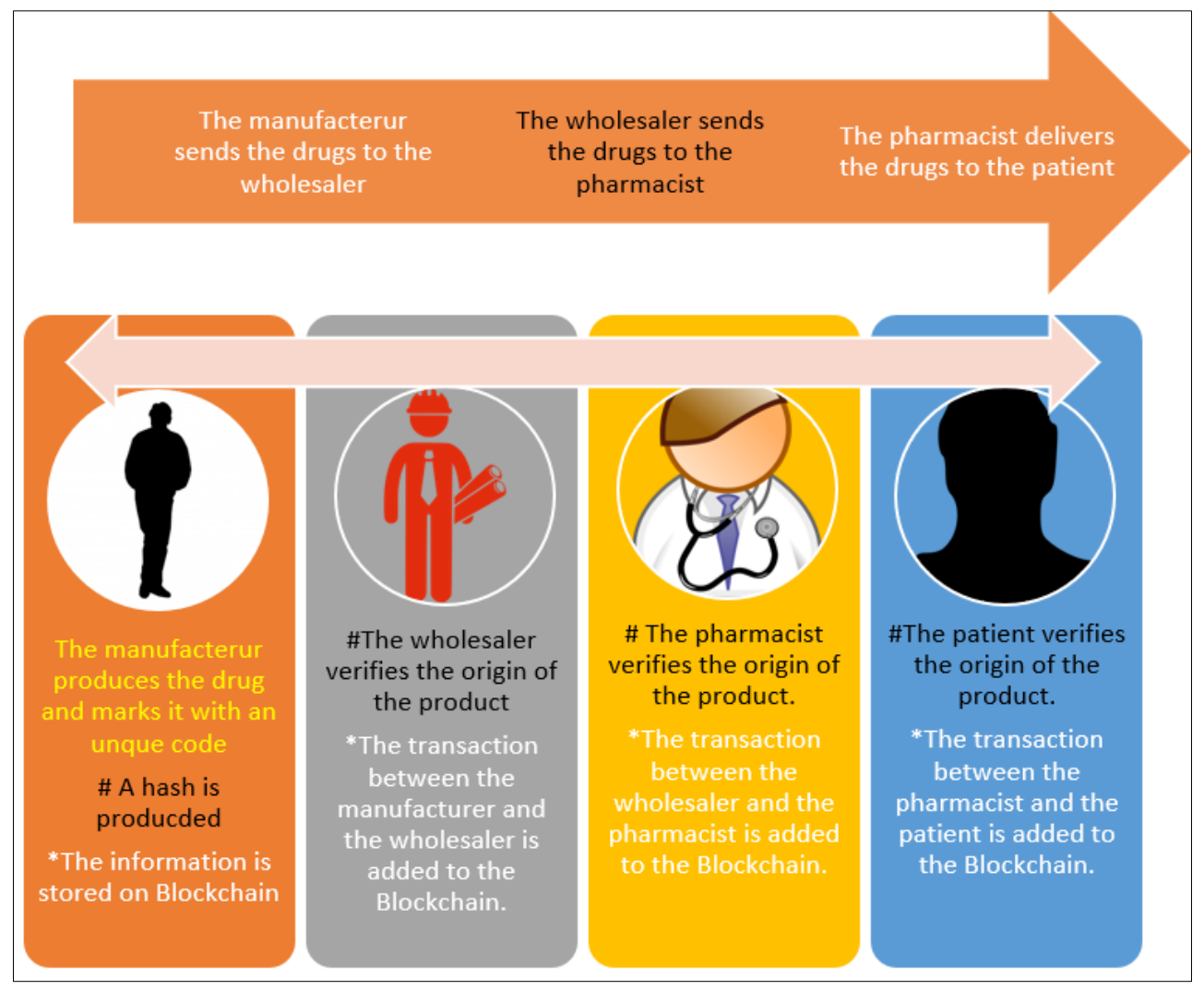

Figure 5 Blockchain for drug trackability

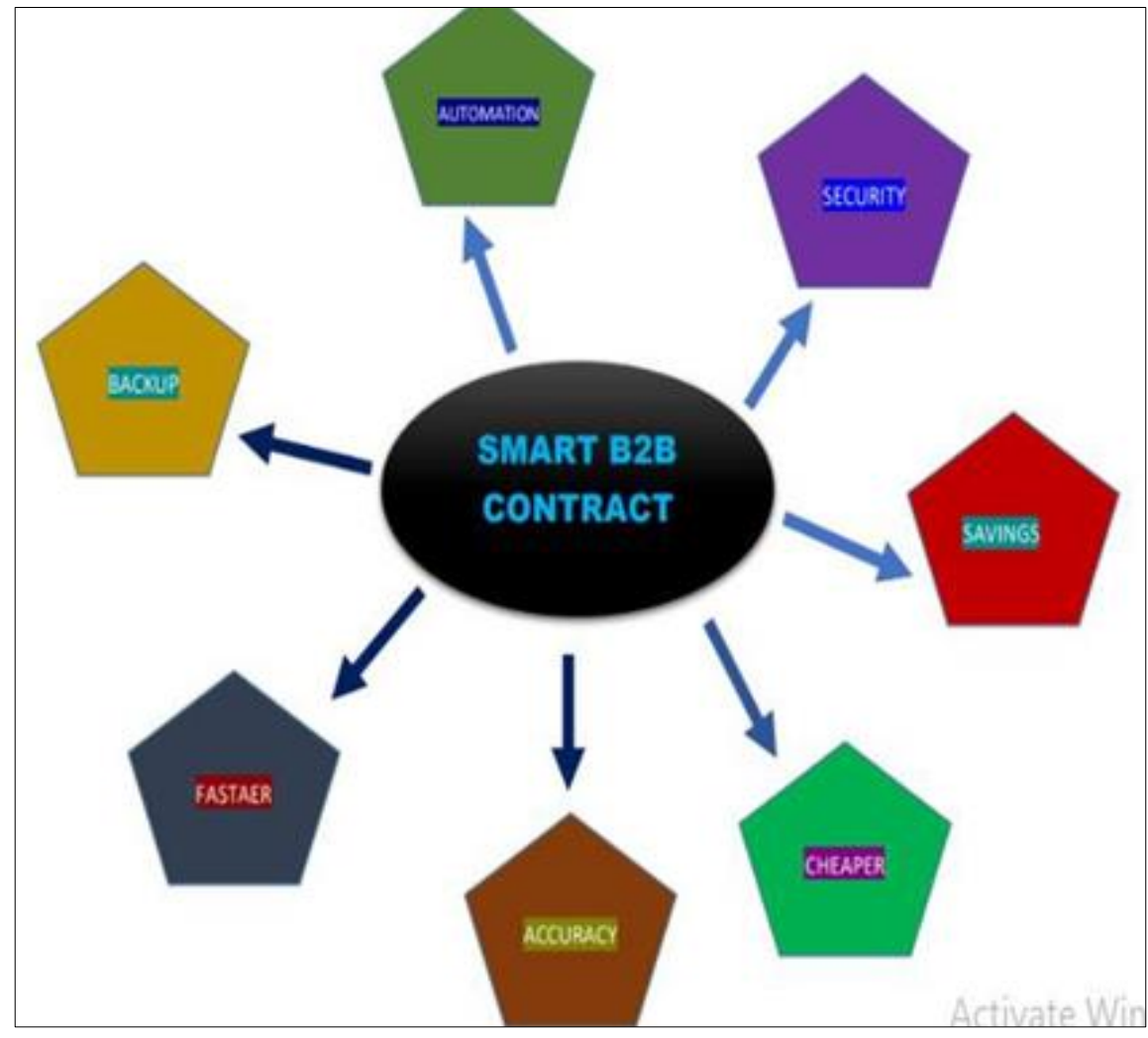

Figure 6 Smart B2B contract 
Smart contract required fill up the form from top so there is no possibility of error and it is cheaper and faster. Via smart contract all documents are encrypted so trust issue problem removed. Another thing of smart contract is it is client can add data after matching the rules which are already created earlier. The most pleasant thing about smart contract is the Ethereum project. This project working as same as bitcoin means to store all transaction record but different thing is it helps to keep track of the state and also network keep track of current information. Via this network developer can apply code into the smart contract using high-level language. Aptly, Tipco, CVS- Aetna companies are able to create smart b2b contract already through Intelligent Healthcare Blockchain Technology to provide secure, hassle-free, verified payment processing $[4$

\subsection{Fill up forms for hospital intake}

In hospital usually people fill up the form manually for security purposes using queue and wasting time. But blockchain technology gives us opportunity to fill up this form in a second and patients can avoid line and save their time. The firm calibrate blockchain recently developed blockchain-powered engine named Universal Patient Index and it is basically Form Drop Mobile App. This app helps a patient to create a form accurately and provides efficiency.

\subsection{Patient data management}

Every patient case is different than another. Patient strategies vary from patient to patient as everyone's health is not similar. A particular disease does not work in the same way for every patient. So a unique structure or common treatment does not help in this case. One treatment that helps one patient might not be helpful for another one.

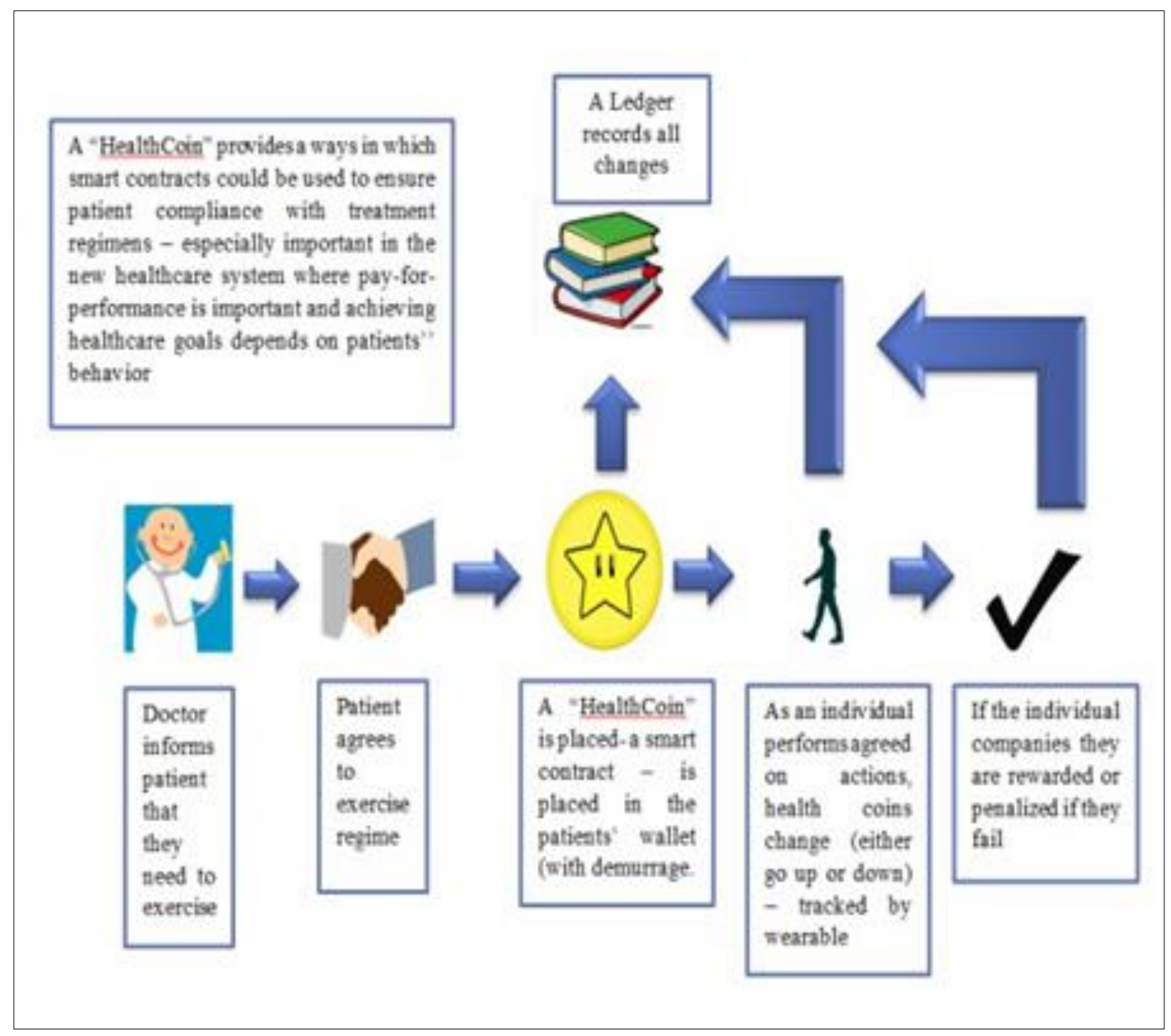

Figure 7 Patient data management 
To give patients personalized care, a doctor needs full details of health records of that particular patient. But in most cases, doctors do not get access to every data of their patients. In medical sector, data sharing is very challenging issue. Especially in case of transferring to a new medical facility, patient has to do all the tests again which costs them a large amount of money. Because patient does not have full access to his/her own medical records. Usually, hospitals do not share every data with patients so that patients can't change or delete any information. They keep the information without patients' permits [5]. Moreover, the patient data storage system is not a safe system. The way doctors share their patient data is very insecure way. They use social media for this purpose. There is a big chance of data leaking in this way. That's why patient data management is a very complex issue in today's world. Blockchain gives us solutions to solve every problem related to patient data management. Blockchain stores all the patient data in a structured way. The patients have complete authority over their medical records only. It is also possible to store the data in a way that only the patient case-handling doctor and his/her patient can access them. Patients also can cancel the access anytime. Besides, those data which are not sensitive, are accessible publicly for the hospitals and stakeholders. They must access those through an ape system. Thus, collaboration from every different system happens in a secured and structured way [6]. Patients can make their data publicly accessible anonymously. Hospitals and other healthcare organizations can use those for their research purpose. But only a patient has the power to decide with whom he/she wants to share the information. Blockchain uses smart contracts to manage the access of patient data. It is not possible to change or delete any data which wipes off the misuse of data from the medical field. Transferring patient data from one facility to another is very easy with blockchain. Doctors now can easily make the treatment patient centric as they have the full details of their patients to understand the situation and disease properly. When blockchain and IoT are combined, doctors and researchers can make fast decisions by constantly monitoring vital organ function just as heartbeat, which can help to save a critical condition patient. With constant tracking, patients' treatment will be improved. Also, patient has the power to make the causes and consequences of what happens if she/he can't reach them in time. So, we can say that Blockchain certainly can improve the present way of data managing in medical sector and a lot more [7].

\section{Blockchain technology architecture in healthcare}

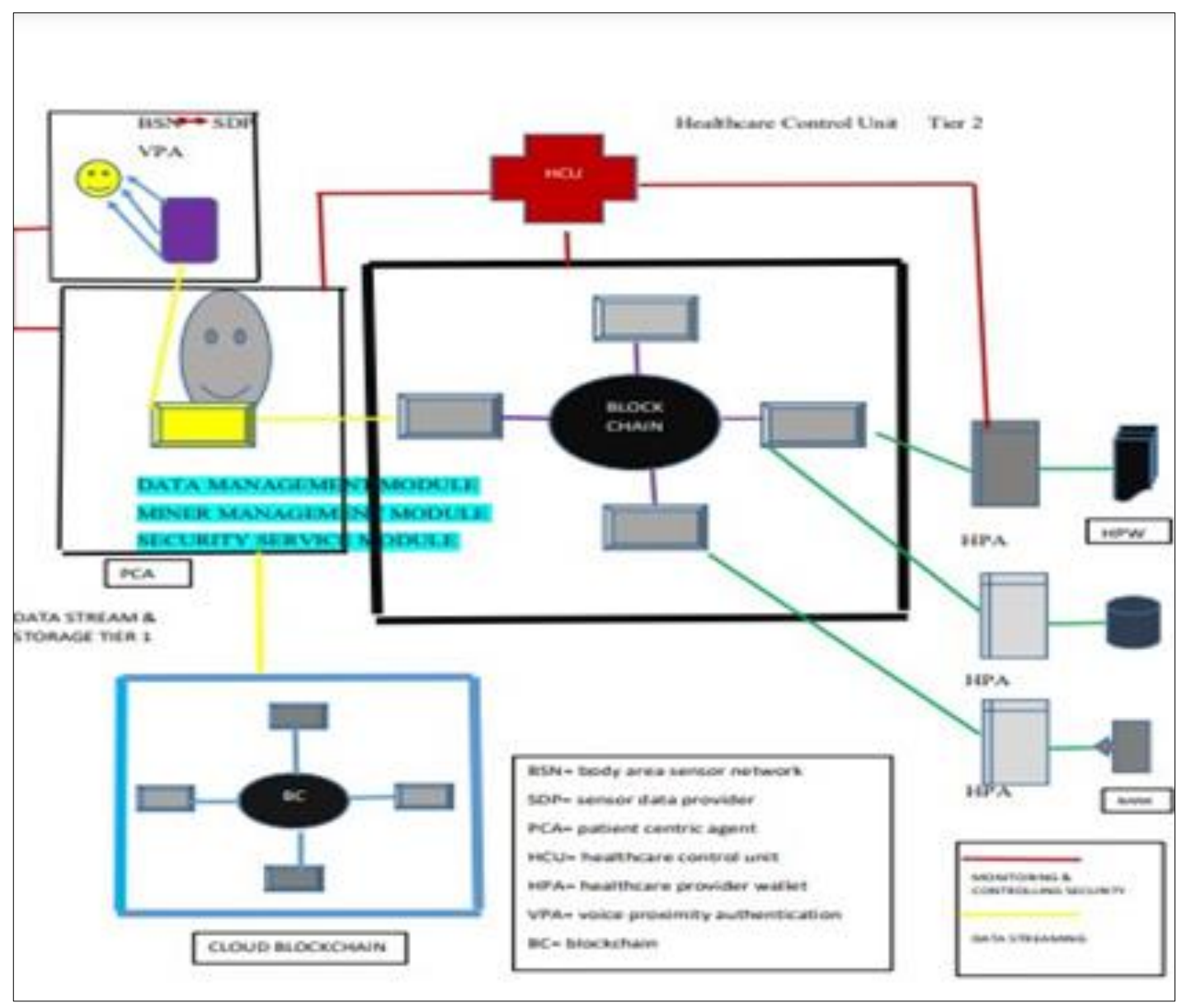

Figure 8 Blockchain technology architecture in healthcare 


\section{Future Scope}

The future Scope of blockchain is stated below: Tracking and authenticating the health care chain to understand provenance can be promised. Solving traceability issues and ensuring transparency can be done by using blockchain. Blockchain can grow disruptive in the Internet of Things. Research suggests that blockchain technology can help to achieve traceability All the promising features of blockchain can be fulfilled. We can create apps on blockchain. We can provide emergency numbers to the villagers in order to use the technology in the rural areas of our country also [8].

\section{Conclusion}

Blockchain technology has a lot of potential for healthcare sector. Blockchain can improve the problems current healthcare industry is facing right now. Though it is in its primary stage, we will be able to see more innovative solutions in the upcoming years. With blockchain technology emerging, the future for medical and pharmaceutical sectors is certainly bright.

\section{Compliance with ethical standards}

\section{Acknowledgments}

The authors expressed their gratitude and appreciation to A K M Bahalul Haque, Lecturer of the Department of Electrical and Computer Engineering of North South University who has helped and supervised them throughout the process.

\section{Disclosure of conflict of interest}

The author(s) declared no potential conflicts of interest with respect to the research, authorship, and/or publication of this article.

\section{References}

[1] Berentsen A. Aleksander Berentsen Recommends "Bitcoin: A Peer-to-Peer Electronic Cash System" by Satoshi Nakamoto. In21st Century Economics. 2019; 7-8.

[2] Meinert E, Alturkistani A, Foley KA, Osama T, Car J, Majeed A, Van Velthoven M, Wells G, Brindley D. Blockchain Implementation in Health Care: Protocol for a Systematic Review. JMIR Research Protocols. 2019; 8(2): e10994.

[3] Esposito C, De Santis A, Tortora G, Chang H, Choo KR. Blockchain: A Panacea for Healthcare Cloud-Based Data Security and Privacy? In: IEEE Cloud Computing. 2018; 5(1): 31-37.

[4] Guo R, Shi H, Zhao Q, Zheng D. Secure Attribute-Based Signature Scheme With Multiple Authorities for Blockchain in Electronic Health Records Systems. In: IEEE Access. 2018; 6: 11676-11686.

[5] Ichikawa D, Kashiyama M, Ueno T. Tamper-Resistant Mobile Health Using Blockchain Technology. JMIR mHealth and uHealth. 2017; 5(7): e111.

[6] Liang X, Shetty S, Zhao J, Bowden D, Li D, Liu J. Towards decentralized accountability and self-sovereignty in healthcare systems. In International Conference on Information and Communications Security. 2017 Dec 6; 387 398.

[7] Mertz L. Hospital CIO Explains Blockchain Potential: An Interview with Beth Israel Deaconess Medical Center's John Halamka. In: IEEE Pulse. 2018; 9(3): 8-9.

[8] Zhang J, Xue N, Huang X. A Secure System for Pervasive Social Network-Based Healthcare. In: IEEE Access. 2016; 4: 9239-9250. 